\title{
Theory of thermopower in 2D graphene
}

\author{
E. H. Hwang, E. Rossi, and S. Das Sarma \\ Condensed Matter Theory Center, Department of Physics, \\ University of Maryland, College Park, Maryland 20742-4111
}

(Dated: August 27, 2018)

\begin{abstract}
Motivated by recent experiments 1, 2, 3] we calculate the thermopower of graphene incorporating the energy dependence of various transport scattering times. We find that scattering by screened charged impurities gives a reasonable explanation for the measured thermopower. The calculated thermopower behaves as $1 / \sqrt{n}$ at high densities, but saturates at low densities. We also find that the thermopower scales with normalized temperature $T / T_{F}$ and does not depend on the impurity densities, but strongly depends on the fine structure constant $r_{s}$ and the location of the impurities. We discuss the deviation from the Mott formula in graphene thermopower, and use an effective medium theory to calculate thermopower at low carrier density regimes where electron-hole puddles dominate.

PACS numbers: 81.05.Uw; 73.61.Ey, 73.50.Jt, 71.30.+h
\end{abstract}

Thermopower has been used as a powerful tool to probe transport mechanisms in metals and semiconductors. Often the measurement of resistivity (or conductivity) is inadequate in distinguishing among different scattering mechanisms and the thermopower can then be used as a sensitive probe of transport properties since it provides complementary information to resistivity. In this Letter we develop a theory for the thermopower of graphene with a goal toward elucidating the comparative importance of various scattering mechanisms in graphene transport properties.

Recently, the thermoelectric properties of graphene have attracted experimental attention [1, 2, 3, 4]. Experimentally [1, 2, 3] the expected change of sign in the thermopower is found across the charge neutral point as the majority carriers change from electrons to holes. Away from the charge neutral region the density dependence of thermopower behaves as $1 / \sqrt{n}$, and exhibits a linear temperature dependence in agreement with the semiclassical Mott formula [5]. As the temperature increases, a deviation from Mott formula is reported [1, 2]. Existing theoretical works on graphene thermopower either use an impurity band model or consider the low temperature Mott limit [6]. In this paper we present a calculation of the thermopower of graphene taking into account the energy dependent scattering time for various scattering mechanisms. Understanding thermopower requires a thorough knowledge of the details of energy dependence of transport scattering times $7,[8,9]$. In metals the Mott formula is widely used because the Fermi temperature is very high (i.e. $T \ll T_{F}$ ) and the scattering time is essentially energy independent leading to a simple linear-in temperature form for thermopower which is proportional to the energy derivative of the conductivity evaluated at the Fermi energy. Mott formula, derived mathematically through the Sommerfeld expansion, is only valid at very low temperatures, $T / T_{F} \ll 1$.

We show that scattering by random charged impurity centers, which is the main scattering mechanism limit- ing graphene conductivity, [10] also dominates its thermopower. We show that the temperature dependent screening effects [11] must be included in the theory to get quantitative agreement with existing experimental data. We find the effects of short-range scattering and phonons to be negligible in experimental temperature range $(T<300 K)$, allowing us to ignore phonon drag contribution. We also find that the calculated thermopower scales with $T / T_{F}$ and manifests no impurity density $\left(n_{i}\right)$ dependence, but depends strongly on the impurity location and the dielectric constant of the substrate (or equivalently the fine structure constant of graphene). The experimentally observed asymmetry between electron and hole thermopower is explained by the asymmetry in the charged impurity configuration in the presence of the gate voltage. We explain the experimentally observed sign change near charge neutral point (Dirac point) with a simple two component model, which we explicitly verify using an effective medium theory calculation taking into account the inhomogeneous puddle formation. [10]

The ratio of the measured voltage to the temperature gradient applied across the sample is known as the Seebeck coefficient (or the thermopower) and is given by $Q=\nabla V / \nabla T$, where $\nabla V$ is the potential difference and $\nabla T$ the temperature difference between two points of the sample [7]. In linear response approximation for the electrical current density, $\mathbf{j}$, and thermal current density, $\mathbf{j}_{Q}$, we have: $\mathbf{j}=L^{11} \mathbf{E}+L^{12}(-\nabla T), \mathbf{j}_{Q}=L^{21} \mathbf{E}+L^{22}(-\nabla T)$, where $L^{i j}$ is defined in terms of the $I^{(\alpha)}$, i.e., $L^{11}=I^{(0)}$, $L^{12}=-\frac{1}{e T} I^{(1)}, L^{21}=-\frac{1}{e} I^{(1)}$, and $L^{22}=\frac{1}{e^{2} T} I^{(2)}$. Here, $I^{(\alpha)}$ is given by

$$
\begin{aligned}
I^{(\alpha)} & =e^{2} g \sum_{\mathbf{k}} \tau\left(\epsilon_{\mathbf{k}}\right) \mathbf{v}_{\mathbf{k}} \mathbf{v}_{\mathbf{k}}\left[\epsilon_{\mathbf{k}}-\mu\right]^{\alpha}\left(-\frac{\partial f_{\mathbf{k}}^{0}}{\partial \epsilon_{\mathbf{k}}}\right) \\
& =\int d \varepsilon(\varepsilon-\mu)^{\alpha}\left(-\frac{\partial f^{0}(\varepsilon)}{\partial \varepsilon}\right) \sigma(\varepsilon),
\end{aligned}
$$

where $f_{\mathbf{k}}^{0}$ is the equilibrium Fermi distribution function, 
$\tau$ the relaxation time, and $\mu$ the chemical potential, $g=$ $g_{s} g_{v}$ is the total degeneracy $\left(g_{s}=2, g_{v}=2\right.$ being the spin and valley degeneracies, respectively), and $\sigma(\varepsilon)$ is the energy dependent conductivity of graphene given by $\sigma(\varepsilon)=e^{2} v_{F}^{2} D(\varepsilon) \tau(\varepsilon) / 2$, where $v_{F}$ is the Fermi velocity and $D(\varepsilon)=g|\varepsilon| /\left(2 \pi \hbar^{2} v_{F}^{2}\right)$ the density of states. From the definition of the thermopower we have $Q=\frac{L^{12}}{L^{11}}$, and, $\sigma=L^{11}$.

Before we calculate the details of the thermopower for different scattering mechanisms we first consider the low temperature and high temperature behavior of $Q(T)$. At low temperatures $\left(T \ll T_{F}\right.$, where $\left.T_{F}=E_{F} / k_{B}\right)$ we can express $I^{(\alpha)}$ as

$$
I^{(\alpha)}=\frac{1}{4 \beta^{\alpha}} \int_{-\infty}^{\infty} d x \frac{x^{\alpha}}{\tanh ^{2}(x / 2)}\left[\sigma(\mu)+\left.\frac{x}{\beta} \frac{\partial \sigma(\varepsilon)}{\partial \varepsilon}\right|_{\varepsilon=\mu}\right]
$$

where $\beta=1 / k_{B} T$. Thus we have the well-known Mott formula [5] of thermopower at low temperatures, i.e.

$$
Q=-\left.\frac{\pi^{2}}{3 e} \frac{T}{\sigma(\mu)} \frac{\partial \sigma(\varepsilon)}{\partial \varepsilon}\right|_{\varepsilon=\mu} .
$$

If the energy dependence of the relaxation time is unimportant the sign of the thermopower is determined by whether the carriers are electrons or holes. Assuming the energy dependent scattering time to be $\tau \propto \varepsilon^{m}$ we have the thermopower at low temperatures

$$
Q=-\frac{\pi^{2}}{3 e} \frac{k_{B} T}{T_{F}}(m+1) .
$$

We note that in general the exponent ' $m$ ' has weak temperature and density dependence since $\tau$ behaves only as an effective power law in energy. Eq. (41) indicates that the thermopower can change sign if $m<-1$. At high temperatures $\left(T \gg T_{F}\right)$ we can express the $I^{(\alpha)}$ with an energy dependent scattering time $\tau=\tau_{0} \varepsilon^{m}$ as

$I^{(\alpha)} \approx E_{F}^{\alpha}\left(\frac{T}{T_{F}}\right)^{\alpha+m+1}\left[1-\frac{1}{2^{\alpha+m}}\right] \Gamma(\alpha+m+2) \zeta(\alpha+m+1)$,

where $\Gamma(z)$ and $\zeta(z)$ are the gamma function and Riemann's zeta function, respectively, from which we find:

$$
Q \approx \frac{k_{B}}{e} \frac{(m+2)}{2} \frac{\left(2^{m+1}-1\right)}{\left(2^{m}-1\right)} \frac{\zeta(m+2)}{\zeta(m+1)} .
$$

At high temperatures $Q$ in graphene approaches a limiting value. In Fig. 11 we show the calculated graphene thermopower for different scattering exponents $m\left(\tau \propto \varepsilon^{m}\right)$ as a function of $T / T_{F}$. As shown in Fig. 1 the dashed lines representing Mott formula agree with the full calculations for $T \lesssim 0.2 T_{F}$. In addition the calculated thermopower scales as a function of the normalized temperature $\left(T / T_{F}\right)$.

Now we calculate the thermopower in the presence of various physical scattering mechanisms. For both neutral

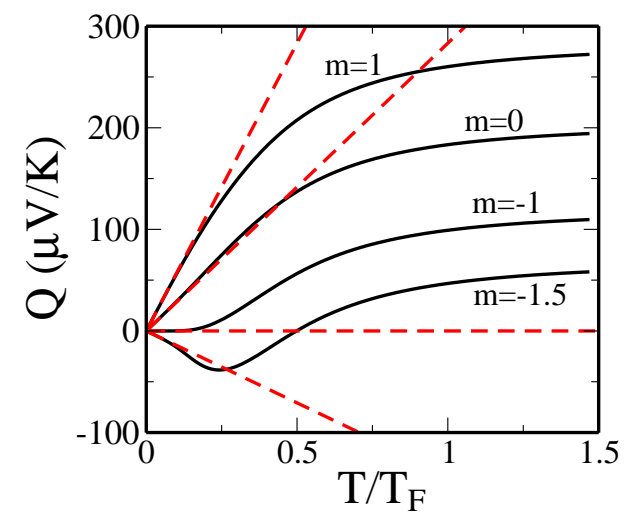

FIG. 1: Hole thermopower for different energy dependent scattering times, $\tau \propto \varepsilon^{m}$. For $m<-1$ the low temperature thermopower becomes negative. Dashed lines show the Mott formula for the corresponding scattering times. Electron results are the same with an overall negative sign.

white noise short-range disorder and acoustic phonon [12] scattering we can express the scattering time as $\tau(\epsilon)=$ $\tau_{1} / \epsilon$, and we have

$$
\begin{aligned}
I^{(0)} & =\sigma_{1} \frac{1}{1+e^{-\beta \mu}}, \\
I^{(1)} & =\sigma_{1}\left[k_{B} T \ln \left[1+e^{-\beta \mu}\right]+\frac{\mu}{1+e^{\beta \mu}}\right] .
\end{aligned}
$$

Then the thermopower becomes

$$
Q=-\frac{1}{e}\left[\frac{\mu}{T} e^{-\beta \mu}+O\left(e^{-\beta \mu}\right)\right] .
$$

The thermopower contributions from both neutral scatterers and acoustic phonons are exponentially suppressed in the low temperature limit, and can be ignored. For unscreened charged impurities we have very simple energy dependent scattering time [11] $\tau(\epsilon)=\tau_{0} \epsilon$. Then we have the following integrals for $t \ll 1$

$$
\begin{aligned}
& I^{(0)}=\sigma_{0}\left[1+O\left(e^{-\beta \mu}\right)\right], \\
& I^{(1)}=\sigma_{0} E_{F}\left[\frac{2 \pi^{2}}{3} t^{2}+O\left(e^{-\beta \mu}\right)\right],
\end{aligned}
$$

leading to the thermopower

$$
Q=-\frac{2 \pi^{2}}{3 e} \frac{k_{B}^{2} T}{E_{F}}\left[1+O\left(e^{-\beta \mu}\right)\right] .
$$

The linear relation of thermopower with temperature (or Mott formula) holds to relatively high temperatures in graphene for unscreened Coulomb impurities.

As has been demonstrated theoretically and experimentally the dominant transport mechanism in graphene is the screened Coulomb scattering from charged impurities. The result of Eq. (12) for unscreened Coulomb scattering is much higher than the thermopower observed 
in experiments [1, 2, 3] and cannot explain the behavior of $Q$ close to the Dirac point. An accurate quantitative agreement between theory and experiment can only be achieved by taking into account the screening of the charged impurities and the strong spatial inhomogeneity that these impurities induce close to the Dirac point. For the screened charged impurity scattering, the energy dependent scattering time $\tau\left(\varepsilon_{k}\right)$ is given [10]

$$
\begin{aligned}
\frac{1}{\tau\left(\epsilon_{k}\right)}= & \frac{\pi n_{i}}{\hbar} \int \frac{d^{2} k^{\prime}}{(2 \pi)^{2}}\left|\frac{v_{i}(q)}{\epsilon(q, T)}\right|^{2} \delta\left(\epsilon_{\mathbf{k}}-\epsilon_{\mathbf{k}^{\prime}}\right) \\
& \times(1-\cos \theta)(1+\cos \theta),
\end{aligned}
$$

where $\theta$ is the scattering angle, $v_{i}(q)=$ $2 \pi e^{2} \exp (-q d) /(\kappa q)$ is the Fourier transform of the 2D Coulomb potential in an effective background lattice dielectric constant $\kappa$ and $d$ is the location of charged impurity measured from graphene surface. In Eq. (13), $\varepsilon(q) \equiv \varepsilon(q, T)$ is the $2 \mathrm{D}$ finite temperature static RPA dielectric (screening) function appropriate for graphene [11], given by $\varepsilon(q, T)=1+v_{c}(q) \Pi(q, T)$, where $\Pi(q, T)$ is the graphene irreducible finite-temperature polarizability function and $v_{c}(q)$ is the Coulomb interaction. There is an important direct $T$ dependence of thermopower, not captured in the Mott formula, arising from the temperature dependent screening. [11] The temperature dependent conductivity due to screening effects decreases quadratically at low temperatures [11]. This mechanism produces a thermopower quadratic in temperature rather than linear as in the simple Mott formula. Thus we predict a nonlinear quadratic temperature correction in the graphene thermopower compared with the linear Mott formula.

In Fig. 2(a) we show the calculated thermopower of holes in graphene due to different scattering mechanisms. The thermopower due to screened charged impurity is about half of that due to the unscreened charged impurity, and increases in a concave manner due to temperature and energy dependent screening. On the other hand, the thermopower due to neutral scatterers is exponentially suppressed in the low temperature regime. Fig. 2(b) shows the calculated thermopower for the screened charged impurity scattering as a function of density for different temperatures. As we expect the density dependence shows $1 / \sqrt{n}$ behavior at high densities. But this power law behavior breaks down and saturates at low densities. The saturation value $\left(Q_{s}\right)$ does not depend on temperature. The theoretical $Q_{s}$ is just a function of interaction (fine structure) parameter $r_{s}=e^{2} / \kappa \hbar v_{F}$ and the location of impurities $d$. In Fig. 2 (c) and (d) we show the $r_{s}$ and $d$ dependence of the thermopower. In general the thermopower increases when the substrate dielectric constant $(\kappa)$ increases or the charged impurities move away. Thus, we predict that the thermopower of suspended graphene will decrease compared with the thermopower of graphene on a substrate for the
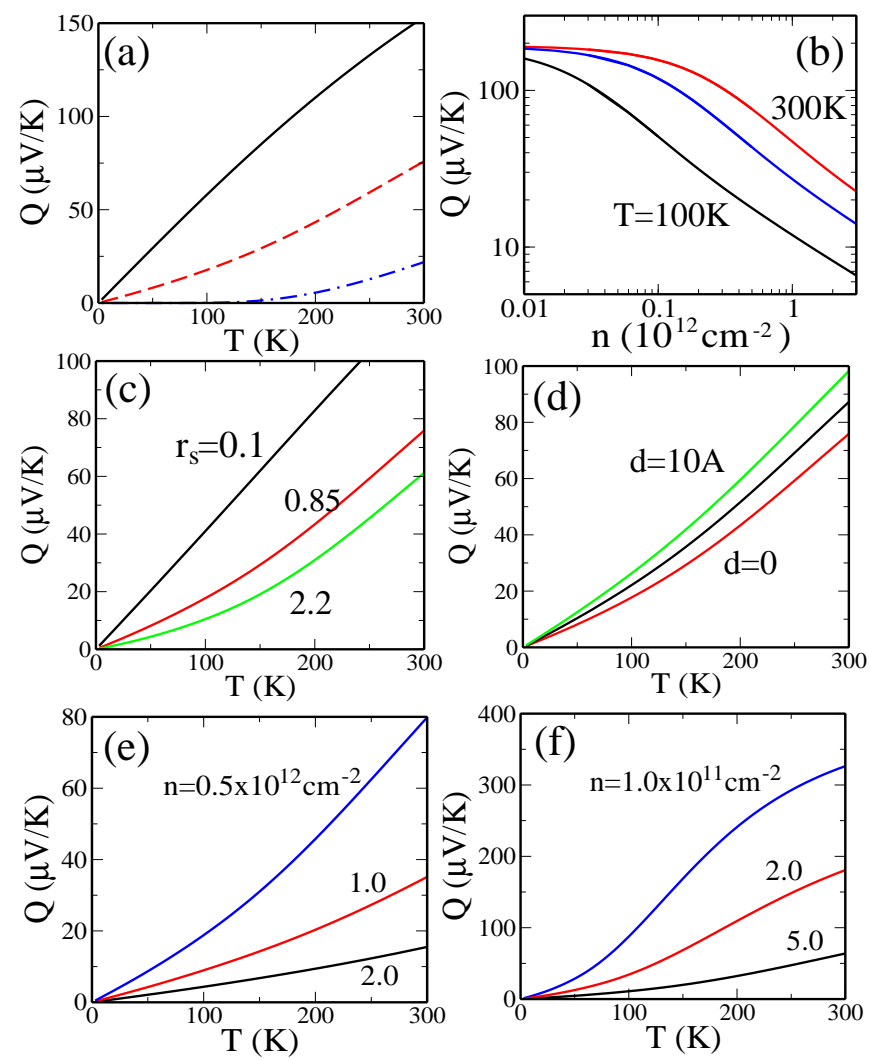

FIG. 2: (a) $Q$ as a function of temperature for different scattering mechanisms. Solid, dashed, and dot-dashed line represent unscreened Coulomb, screened Coulomb, and neutral scatterers, respectively. (b) $Q$ for the screened charged impurity scattering as a function of density for different temperatures. (c) $Q$ for different $r_{s}=0.1,0.85,2.2$ with $d=0$ and (d) for different $d=0,5,10 \AA$ with $r_{s}=0.85$. In (e) and (f), $Q$ in the presence of screened charged impurities for different densities is shown. In (e) we use parameters corresponding to graphene on $\mathrm{SiO}_{2}$ with mobility $\mu=10^{4} \mathrm{~cm}^{2} / \mathrm{Vs}$, and in (f) to suspended graphene with mobility $\mu=2 \times 10^{5} \mathrm{~cm}^{2} / \mathrm{Vs}$.

same densities because of the reduction of the dielectric constant. In Fig 2 (e) and (f) we show the calculated thermopower for systems with different mobilities due to screened charged impurity scattering for graphene on $\mathrm{SiO}_{2}$ substrate and for suspended graphene.

In recent experiments [1, 2, 3] it has been observed that close to the Dirac point $Q$ does not follow the $1 / \sqrt{n}$ scaling predicted by the Mott formula. The reason for this deviation is that close to the Dirac point the quenched disorder induces strong density fluctuations that break up the density landscape in electron-hole puddles [10, 13, 14, 15]. To account for the main features of the thermopower close to the Dirac point we can use a simple two component model near to the charge neutral regime in which the electron density, $n_{e}$ and the hole density $n_{h}$ depend on the doping $n$ according to the phenomenological equations: $n_{e}=\left(n_{\mathrm{rms}}+n\right) / 2$, 
$n_{h}=\left(n_{\mathrm{rms}}-n\right) / 2$ for $|n| \leq n_{\mathrm{rms}}$, where $n_{\mathrm{rms}}$ is the root mean square of the density fluctuations. We assume $n_{\mathrm{rms}} \sim n_{i} 10,14$. In the two component model the thermopower becomes $Q=\left(L_{e}^{12}+L_{h}^{12}\right) /\left(L_{e}^{11}+L_{h}^{11}\right)$ so that, for unscreened charged impurities, we find:

$$
Q=-\frac{k_{B}^{2}}{e} \frac{2 \pi^{2}}{3} \frac{T}{v_{F} \sqrt{\pi}}\left[\frac{\sqrt{n_{e}}-\sqrt{n_{h}}}{n_{e}+n_{h}}\right] .
$$

Thus, if there is an equal number of electrons and holes the thermopower goes to zero, and the overall sign is decided by the majority carriers. We find that the thermopower due to the screened charge impurities shows the same behavior as shown by the dashed line in Fig. 3 . In order to explicitly verify the electron-hole puddle picture near the charge neutral point we use the effective medium theory, EMT for graphene [15]. The density profile close to the Dirac point is quantitatively described by the Thomas-Fermi-Dirac (TFD) theory [14]. Using the TFD results the transport properties of graphene close to the Dirac point can be accurately calculated using the EMT. Denoting by angle brackets disordered averaged quantities, for the diagonal transport coefficients from the EMT in 2D we have that the effective medium coefficients, $L_{\text {eff }}^{i i}$, are implicitly given by the equation [16]:

$$
\left\langle\frac{L^{i i}(\mathbf{r})-L_{\text {eff }}^{i i}}{L^{i i}(\mathbf{r})+L_{\text {eff }}^{i i}}\right\rangle=0 .
$$

Adapting to $2 \mathrm{D}$ thermopower the results presented in [17], the effective medium off-diagonal coefficient $L^{12}$ is given by:

$$
\begin{aligned}
& L_{\text {eff }}^{12}=-2 L_{\text {eff }}^{11} L_{\text {eff }}^{22}\left\langle\frac{L^{12}(\mathbf{r})}{\left(L^{11}(\mathbf{r})+L_{\text {eff }}^{11}\right)\left(L^{22}(\mathbf{r})+L_{\text {eff }}^{22}\right)}\right\rangle \times \\
& \left\langle\frac{L^{11}(\mathbf{r}) L_{\text {eff }}^{22}+L_{\text {eff }}^{11} L^{22}(\mathbf{r})+L_{\text {eff }}^{11} L_{\text {eff }}^{22}-L^{11}(\mathbf{r}) L^{22}(\mathbf{r})}{\left(L^{11}(\mathbf{r})+L_{\text {eff }}^{11}\right)\left(L^{22}(\mathbf{r})+L_{\text {eff }}^{22}\right)}\right\rangle^{-1}
\end{aligned}
$$

As shown in [15] for the local values of $L^{i j}$ we can use $L^{i j}(n(\mathbf{r}))$ and then using Eqs. (15), (16) and the probability distribution given by the TFD theory we can calculate the EMT transport coefficients, and in particular the effective medium thermopower $Q_{\text {eff }}=L_{\text {eff }}^{12} / L_{\text {eff }}^{11}$ for graphene at, and away from, the Dirac point. The results for $Q$ as a function of $n$ at $T=300 \mathrm{~K}$ for $r_{s}=0.8$, $d=1 \mathrm{~nm}$ and $n_{i}=10^{12} \mathrm{~cm}^{-2}$ are shown by the solid lines in Fig. 3 in blue (red) are the results obtained with (without) the effect of the exchange term on the density distribution. we note that the two-component model is excellent in describing the main features of the realistic EMT $Q(n)$ close to the Dirac point.

In conclusion we have developed a complete theory for the diffusive thermopower of 2D graphene. Quantitative agreement between our theory and existing graphene experimental thermopower data is a strong indication that the dominant carrier scattering mechanism operational in

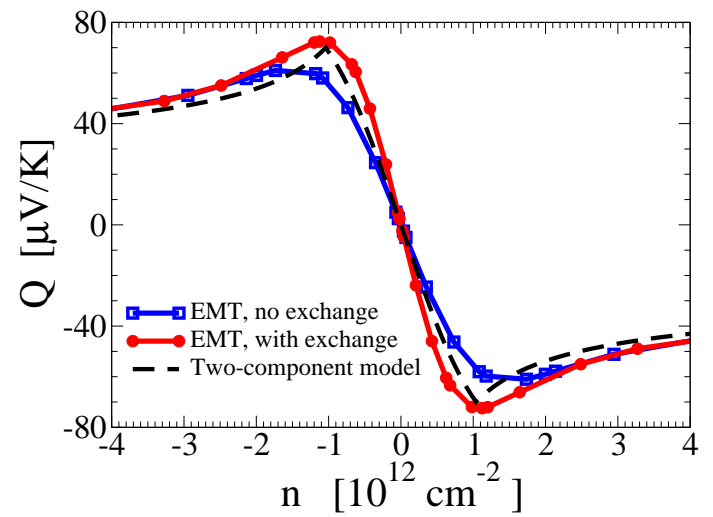

FIG. 3: $Q$ due to the screened charge impurities as a function of density close to the Dirac point for $T=300 \mathrm{~K}$, $n_{i}=10^{12} \mathrm{~cm}^{-2}, r_{s}=0.8$ and $d=1 \mathrm{~nm}$, obtained using the two-component model and the EMT with and without exchange energy.

2D graphene monolayers is screened Coulomb scattering by random charged impurities located in the graphene environment. At high densities the Mott formula applies well to the measured thermopower because of the high Fermi temperature, but it fails in low density limit. We explain the sign change of the thermopower in the low density regime by using both a two component model and a realistic effective medium theory, that correctly describes transport in the presence of the strong carrier density inhomogeneities, that characterize the graphene density landscape close to the Dirac point. We make a number of specific predictions for graphene thermopower (e.g. nonlinearity in temperature, existence of a saturation thermopower at low densities, nontrivial dependence on the background dielectric constant and on the impurity location), which should be tested experimentally in order to conclusively settle the issue of dominant carrier scattering mechanism in graphene.

This work is supported by the U.S.-ONR and NSFNRI-SWAN.

[1] Yuri M. Zuev et al., arXiv:0812.1393

[2] Peng Wei et al., arXiv:0812.1411.

[3] Joseph G. Checkelsky and N. P. Ong, arXiv:0812.2866

[4] A.A. Balandin et al., Nano Letters 8, 902 (2008).

[5] M. Cutler and N. F. Mott, Phys. Rev. 181, 1336 (1969).

[6] T. Löfwander and M. Fogelström, Phys. Rev. B 76, 193401 (2007); T. Stauber et al., Phys. Rev. B 76, 205423 (2007); B. Dora and P. Thalmeier, Phys. Rev. B 76, 035402 (2007); M.S. Foster and I.L. Aleiner, Phys. Rev. B 77, 195413 (2008); M. Müller et al., Phys. Rev. B 78, 115406 (2008).

[7] N. W. Ashcroft and N. D. Mermin, Solid State Physics, (Thomson Learning Inc. USA, 1976). 
[8] L. Moldovan et al. Phys. Rev. Lett. 85, 4369 (2000).

[9] S. K. Lyo, Phys. Rev. B 70, 153301 (2004).

[10] E. H. Hwang et al. Phys. Rev. Lett. 98, 186806 (2007); S. Adam et al., Proc. Natl. Acad. Sci. USA 104, 18392 (2007).

[11] E. H. Hwang and S. Das Sarma, arXiv:0811.1212; Phys. Rev. B 75, 205418 (2007).

[12] E. H. Hwang and S. Das Sarma, Phys. Rev. B 77, 115449 (2008).
[13] J. Martins et al., Nature Phys. 4, 144 (2008).

[14] E. Rossi and S. Das Sarma Phys. Rev. Lett. 101, 166803 (2008).

[15] E. Rossi et al., arXiv:0809.1425 (2008); M. Fogler, arXiv:0810.1755 (2008).

[16] D. A. G. Bruggeman Ann. Physik 416, 636 (1935); R. Landauer J. Appl. Phys. 23, 779 (1952).

[17] I. Webman et al. Phys. Rev. B 16, 2959 (1977). 